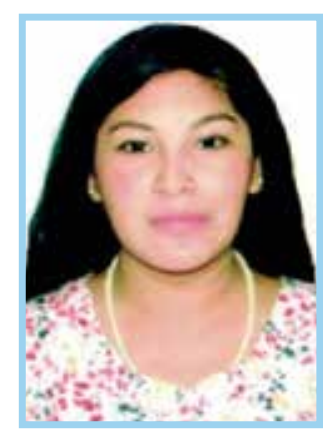

Mayra Yaranga

Hernández

\section{EL INGLÉS COMO SEGUNDA LENGUA: ACCESO Y OPORTUNIDAD EN EL SISTEMA UNIVERSITARIO}

\section{RESUMEN}

A través del presente artículo, se busca profundizar la noción de que el conocimiento de una lengua extranjera como el inglés es necesario para el profesional moderno, noción que a menudo suele carecer de un sustento específico y cae dentro de la categoría de cliché. Por esto, se desarrollarán dos aspectos dentro del contexto de la educación superior universitaria: la necesidad del aprendizaje del inglés entendida como acceso, y el potencial no utilizado de los intercambios académicos internacionales para aprender a vivir en la comunidad global. De esta manera, se pretende brindar una visión más clara y específica sobre las implicancias del aprendizaje del inglés dentro del contexto de la formación universitaria contemporánea.

\section{PALABRAS CLAVE}

Intercambios académicos internacionales, ESP, EAP, certificados internacionales de idiomas.

\section{ABSTRACT \\ Through this article, which is searching to go in depth the idea about a foreign language knowledge as English is needed for the modem professional, that idea usually does not have a specific support and falls within cliché category. That's why two aspects will be develop within the context of university higher education: the English learning necessity understood as access and the potential did not use of the international academic exchanges for learning to life in the global community. In that way, is expecting to give a clearer and specific view about English learning implications within contemporary university education context.}

\section{KEYWORDS}

International academic exchanges, ESP, EAP, language international certificates.

\section{INTRODUCCIÓN}

—n los últimos años, la comunidad universitaria ha experimentado el desarrollo de una actitud positiva hacia el conocimiento de una lengua extranjera, por lo general el inglés: su conocimiento es requisito para la obtención de un grado académico, y por ende el estudiante universitario promedio está concientizado en que el inglés es importante o necesario para la carrera, y que por ende hay que saberlo. Sin embargo, estos tres términos en cursivas pueden ser imprecisos, ya que su verdadera dimensión no es necesariamente conocida o no ha sido definida por las instituciones educativas.

El Segundo Censo Nacional Universitario realizado en 2010 por la Asamblea Nacional de Rectores (ANR) y el Instituto de Estadística e Informática (INEI) 
compartió resultados acerca del nivel de inglés que se muestran más alentadores respecto al primer censo, pues el número de estudiantes de pregrado con conocimiento de inglés aumentó de 30,8\% en 1996 a 43,7\% (CENAUN, 2010). En cuanto a los docentes, un $75,8 \%$ de docentes declara tener conocimiento de inglés respecto a un $74,3 \%$ en 1996 . La cédula censal solo contempla que el encuestado marque si habla, lee o escribe en inglés.

Siconsideramos quelas cifras proporcionadas por esta encuesta se adecuan a la realidad, entonces se podría esperar que, por ejemplo, un gran número de estudiantes o docentes investigadores accedieran a programas de intercambio o becas en universidades de países no hispanohablantes. No obstante, como se verá más adelante, este no es el caso, y muchas oportunidades existentes no son tomadas, siendo la falta de conocimiento del inglés un factor importante para explicar este déficit. Si bien no es posible negar los resultados de la encuesta o calificarlos de inválidos, se debe prestar atención al aparente reduccionismo en el que cae este instrumento: la percepción individual del nivel de dominio de una lengua está afectada por el general desconocimiento de los factores involucrados en el aprendizaje; por esta razón, es posible que una persona sobreestime o subestime su nivel si este no está claramente definido, o si no se somete a una prueba más rigurosa para determinarlo. Evidentemente, no es posible realizar una prueba así para propósitos de un censo, pero si se contara con información oficial de las mismas universidades al respecto, tal vez las cifras representarían de una manera más precisa esta información, basada no en una percepción subjetiva sino en un instrumento objetivo.

En las secciones posteriores, se revisará los aspectos del acceso y la oportunidad que brinda el dominio del inglés como lengua extranjera en el contexto universitario.

\section{ACCESO}

Para entender de manera más profunda la importancia del uso de la lengua inglesa, es preciso tener una perspectiva histórica de su posición en el mundo. En primer lugar, en la actualidad se calcula que existen alrededor de 370 millones de hablantes de inglés como primera lengua (Crystal, 2010) y 1500 millones como segunda lengua. Este número se explica por el desarrollo del Imperio Británico y entre los siglos XVIII y XX, por el posterior posicionamiento de los Estados Unidos en los sectores tecnológico, económico y político desde inicios del siglo $\mathrm{XX} \mathrm{y}$, por último, la necesidad de una lengua franca para el desarrollo de la industria de las comunicaciones y relaciones internacionales. En la actualidad, pese a la crisis económica que atraviesan los EE. UU. y la aparición de una nueva potencia mundial como China, la posición privilegiada de la lengua inglesa se mantiene vigente.

Para el propósito de este artículo, se ha decidido relacionar la necesidad de aprender una lengua como el inglés con la idea de acceso. No es un secreto que, en la actual era del conocimiento, el flujo de información brindada y compartida se encuentra mayoritariamente en inglés. Es por ello que poseer un estándar elevado en el conocimiento de la lengua permitirá encontrar, discriminar, analizar, utilizar y criticar la información difundida a través de medios tradicionales o electrónicos. Asimismo, la importancia de debatir acerca del acceso a la información para vivir en una sociedad más justa no es un tema reciente, pero adquiere urgencia si se tiene como marco principal la significativa expansión de Internet de los últimos años. Un caso particular es el de los Recursos Educativos Abiertos (REA). Uno de los documentos más relevantes respecto a este debate es la Declaración de París de la UNESCO de 2012 sobre los REA, cuyo objetivo principal es promover la difusión de estos recursos para el aprendizaje autónomo 
y gratuito. A pesar de que el documento sugiere promover la adaptación lingüística de estos recursos (traducirlos), es relevante considerar que la gran mayoría de producción intelectual aún se encuentra en inglés, y que la traducción de estos a diversas lenguas locales es un proceso que toma cierto tiempo, considerando a la traducción como una labor que no puede realizarse automáticamente, sino que pasa por etapas desde la revisión del tema por traducir hasta la estandarización terminológica y la revisión por especialistas (Paredes, 2014).

La importancia como acceso también está relacionada con los usuarios de la información, en este caso los estudiantes universitarios de pregrado. Para empezar a analizar la realidad de aquellos con conocimiento de inglés se debe plantear una pregunta: ¿Con qué nivel de inglés llegan los estudiantes a la universidad? Para responder, hay que ubicarse en la etapa previa: los estudios secundarios de la Básica Regular. Existen grandes diferencias entre estudiantes provenientes de escuelas públicas y privadas, que en general se deben a la frecuencia de instrucción. Por un lado, los colegios públicos, según el Diseño Curricular Básico - DCN (2009), están obligados a incluir dos horas pedagógicas (90 minutos) de inglés durante cinco años, empezando en el primer año de secundaria, pues el nivel de primaria no lo exige. Aun así, hay horas de libre disposición que tanto el nivel primario (10 horas pedagógicas) como secundario (4 horas pedagógicas) podrá designar para la mayor frecuencia de sus lecciones, que de manera óptima llevaría a los colegios a enseñar un máximo de 4 horas pedagógicas. Por el contrario, en el sector privado el sistema es diferente y quizá aquí se encuentre la primera gran desventaja en cuanto a la preparación para obtener becas en el extranjero, por ejemplo. Los colegios privados destinan más horas al dictado del inglés, o se proponen a enseñarlo desde el nivel Inicial, sumado a los exámenes internacionales que toman al término de cada nivel y los programas de Bachillerato Internacional (IB) que requieren el dictado de cursos de ciencias y letras en inglés.
En comunicación personal con la Mg. Maria Luisa Salleres, ex Directora del Área de Inglés del colegio San Silvestre, ella expone que el esfuerzo de los estudiantes al llevar inglés desde muy pequeños y alcanzar estándares internacionales a través de certificaciones evidencia sus frutos cuando estudiantes provenientes del colegio en mención pueden usar, sin ninguna dificultad, el inglés para consultar bibliografía e incluirla en sus trabajos de investigación universitarios o cuando, gracias a sus programas de IB, pueden estudiar una carrera de pregrado en universidades extranjeras.

En relación a los procesos universitarios de admisión, se conoce que la mayoría de universidades no considera el conocimiento del idioma inglés como requisito, a excepción de dos casos. Por un lado, la Pontificia Universidad Católica del Perú consideraba hasta el año 2008 preguntas de gramática, vocabulario y comprensión lectora. Un caso más reciente es el de la Universidad Nacional de Ingeniería, que en el año 2013 decidió incluir siete preguntas de inglés en su examen de admisión y de esta manera elevar la rigurosidad a los 5.522 postulantes que deseaban alcanzar una de las 585 vacantes (Diario La República). Asimismo, Luis Soldevilla del Prado, de la Oficina Central de Admisión de la UNI, en declaraciones al mencionado diario, afirmó que como la mayoría de información publicada para ingenieros se encuentra en inglés, es apropiado que los estudiantes dominen esta lengua (cursivas de la autora).

"La idea es que cada vez tenga mayor exigencia. Ahora estamos empezando con un nivel básicointermedio. A partir de esto, la preparación de los colegios y las academias se tendrán que acomodar para cumplir con todas nuestras exigencias" (Diario La República, 2013).

La acotación que realiza Soldevilla hace una referencia indirecta a la problemática de la preparación en la lengua inglesa, tema desarrollado en párrafos anteriores, y se puede afirmar que la intención original es positiva 
y alentadora. Sin embargo, la inclusión de preguntas en los exámenes de admisión no está libre de cuestionamiento. En primer lugar, se sabe que los estudiantes llegan con niveles disparejos por la diferencia arriba mencionada en la formación lingüística de la escuela. Por otro lado, la mayoría de universidades no contempla el verdadero nivel de los estudiantes ingresantes para orientarlos a mejorarlo en los primeros años de estudio. Por esta razón, sería conveniente realizar una evaluación oral y escrita a todos los alumnos ingresantes, para determinar de manera más precisa su grado de conocimientos; de acuerdo con los resultados, se podría crear cursos de nivelación para que, al llegar a un ciclo determinado antes de la mitad de la carrera, todos los alumnos hayan alcanzado un nivel óptimo en el uso de esta herramienta, que garantizará el acceso, tal como se explicó anteriormente, a la información y a las oportunidades de estudio en el extranjero.

Igualmente, el acceso a información académica incentiva el empezar o continuar trabajando en el campo de la investigación. La búsqueda de fuentes y el entender su contenido y relevancia dentro de un proceso investigativo son aspectos cruciales, y en muchos casos la bibliografía no ha sido traducida, o es de una creación muy reciente, lo que impide encontrar comentarios o resúmenes en castellano. Por ello, el conocer la lengua de publicación supone un ahorro de tiempo y esfuerzo para utilizar la información. Debe suponerse también que el investigador poseerá un grado de conocimientos lingüísticos que le permita obtener el mayor provecho posible de la publicación, y sobre todo entenderla cabalmente, ya que en muchos casos la mala comprensión del discurso académico puede generar fallas dentro de la investigación.

\section{OPORTUNIDAD}

El Informe Delors (1996) contempla cuatro pilares de la educación para la sociedad del siglo XXI. El tercer pilar sugiere aprender a vivir con los demás. Aquí se desea reconocer al otro a través del conocimiento y la valoración de uno mismo para así emprender proyectos en conjunto. Si se traslada este principio al plano universitario, se entenderá la importancia de que los estudiantes universitarios conozcan realidades, estilos de vida y maneras de pensar diferentes a las que encontramos en nuestro país. Para este fin, la internacionalización es un proceso crucial:

La internacionalización de la educación superior es un proceso de transformación institucional por el que los Sistemas Universitarios y sus Instituciones, deciden integrarse y formar parte de la/una comunidad internacional. Para esto deben asumir los estándares y las prácticas de sus pares y homogenizar sus procesos académicos $y$ administrativos, sus títulos y diplomas, para contribuir con la movilidad de personas, programas e ideas. Es decir, que pasan de actuar en un ámbito local a un entorno internacional. (Castillo, 2012).

Al respecto, la Dirección General de Relaciones Internacionales y Cooperación (DGRIC) de la Asamblea Nacional de Rectores, en su Primer Taller de Internacionalización para las Universidades Peruanas (2012), informa que a pesar de algunos ejemplos positivos en el proceso de la internacionalización universitaria, la gran mayoría de ellas carecen de políticas claras. Dentro de los puntos a trabajar, se señala que la competencia de lenguas extranjeras es un punto que aún merece mayor atención. Por un lado, el Informe de Internacionalización (2010) señala que el 72\% del personal de las Oficinas de Relaciones Internacionales de las Universidades Peruanas tiene un nivel bajo o deficiente de lenguas extranjeras y que el 28\% de universidades que cuentan con profesionales cuyo nivel de lenguas extranjeras es eficiente, son privadas y se encuentran en la capital.

Por otro lado, es importante considerar los resultados de participación en programas de intercambio y la postulación a becas en países de habla inglesa. En el primer caso, un 95,6\% de estudiantes de pregrado sostiene que no ha participado en programas de intercambio 
universitario (CENAU, 2010), lo que puede estar ligado a que el proceso de Internacionalización de las universidades peruanas es reciente. Un panorama más revelador se encuentra en la obtención de becas en el extranjero. PRONABEC, a través de las Becas Presidente de la República para realizar estudios de posgrado en el exterior, muestra que la mayoría de estudiantes obtiene más becas para realizar estudios de posgrado en países hispanohablantes en comparación a los angloparlantes (2013). Esto podría estar relacionado a los requisitos que estas últimas consideran antes de proporcionar la carta de aceptación: entre otros, contar con un nivel de inglés avanzado avalado únicamente por exámenes internacionales de dos o tres entidades de prestigio. Aunque la gran mayoría de universidades exige el dominio de un idioma al término de los 5 años de estudios universitarios aceptando certificaciones de sus propios centros de idiomas o de centros autorizados por la ANR, un punto generalmente ignorado es que falta una definición estándar de lo que ciertos niveles quieren decir para diferentes instituciones; en términos prácticos, esto podría significar que lo que para una escuela de idiomas es un nivel intermedio superior, para otra es un nivel básico. Para evitar estos inconvenientes, las universidades podrían impulsar la evaluación bajo exámenes internacionales estandarizados, tal como los exámenes de la Universidad de Cambridge (Gran Bretaña) o la Universidad de Michigan (EE.UU.). Estas evaluaciones representan una gran ventaja pues no tienen caducidad y pueden medir el avance dentro del rango aceptado internacionalmente en niveles de dominio de lenguas europeas, conocido como el Marco Común Europeo de Referencia (MCER).

Por otro lado, las becas para realizar estudios de posgrado ya no se ofrecen únicamente en países de habla inglesa como Gran Bretaña, Estados Unidos, Canadá, Australia o Nueva Zelanda, sino también en países asiáticos como China, India o Corea del Sur, lo que ofrece una oportunidad diferente de ingresar en un sistema educativo de alto rigor académico. Un ejemplo de esta nueva tendencia es el Servicio Alemán de Intercambio Académico (DAAD), organización que incluye a universidades e instituciones de educación superior de Alemania y cuenta con un presupuesto anual de aproximadamente 400 millones de euros, considerado la institución más grande que promueve la movilidad universitaria. La mayoría de becas integrales que ofrece esta organización son para cursos de postgrado dictados en inglés, y sus características son muy ventajosas para los postulantes aptos.

Una manera de fomentar el conocimiento del inglés que es bastante difundida en las universidades peruanas es la de incluir cursos de inglés especializado en las mallas curriculares. Esta es una iniciativa útil que podría ayudar a los estudiantes a familiarizarse con la terminología e incluso con el discurso especializado (English for Specific Purposes ESP) dentro de sus carreras, lo que les da una ventaja competitiva para cuestiones académicas o profesionales. Sin embargo, es posible que si se fomentara entre los estudiantes el alcanzar un nivel óptimo de comprensión y uso de la lengua inglesa, estos cursos de ESP podrían apuntar a un nivel mayor de exigencia para acercarse más a las verdaderas demandas comunicativas del mundo globalizado. Asimismo, la vertiente del inglés para propósitos académicos (English for Academic Purposes - EAP) sería de especial interés para aquellos interesados en acceder a los programas de intercambio, movilidad o las becas descritas anteriormente; los exámenes internacionales son solo el primer paso, ya que el uso de la lengua que evalúan no siempre tienen un matiz académico, y esta familia de géneros discursivos no necesariamente se practica en el sistema universitario peruano. Por otro lado, no es común encontrar en el mercado peruano instituciones que brinden cursos en EAP, lo que manifiesta un vacío en las posibilidades locales de formación en lengua extranjera.

Fuera del contexto de la movilidad universitaria, aprender lenguas extranjeras contribuye con el desarrollo personal. Por un lado, las destrezas 
cognitivas, puesto que ayuda a mejorar las capacidades de comprensión y producción lingüística en la lengua materna. Las estrategias que una persona no ha aprendido o no ha reforzado en su lengua materna, pueden reaprenderse o re-desarrollarse al aprender una segunda lengua. Un estudio comparativo (Dumas, 1999) demuestra que de un total de 13,200 niños, aquellos que recibían instrucción en lenguas extranjeras se desenvolvieron mejor en el test de habilidades básicas respecto a los niños que no tenían esta experiencia. Es sabido que, en muchos casos, aprender diversos aspectos morfosintácticos o léxicos de una lengua pueden ayudar a compararla con la lengua materna, lo que puede redundar en un mejor conocimiento de ambas. No obstante, algo que podríamos considerar incluso más valioso es que el aprendizaje de una segunda lengua promueve la concientización cultural. La realidad en la que vivimos tiende a brindarnos una visión muy sesgada del otro, por lo que el contacto con una lengua extranjera contribuirá con el desarrollo de una actitud solidaria y tolerante hacia los hablantes de dicha lengua y su cultura, lo que podría preparar actitudinalmente al estudiante para poder integrarse en una sociedad distinta, en el caso de que lograra viajar al extranjero. En cualquier caso, el acercamiento a una realidad diferente tiene un efecto notable en el desarrollo de la persona y puede ser considerado un elemento necesario en la formación profesional actual, con las miras de consolidar la identidad nacional en una sociedad pacífica, inclusiva e igualitaria, lo que reflejaría el pilar del informe Delors mencionado al inicio de esta sección.

\section{CONCLUSIONES}

En el marco de la educación universitaria para el presente siglo, es necesario iniciar un proceso orientado a las siguientes metas:

1. Que los actores de la Comunidad Universitaria puedan realizar intercambios académicos y enriquecerse de conocimientos académicos, culturales y para la vida.

2. Que los docentes tengan la oportunidad de incluir textos en esta lengua en sus referencias, o usarlos como material de trabajo.

3. Que tanto docentes como estudiantes puedan asistir a conferencias internacionales y sean capaces de entender de manera eficaz la información brindada.

Por ende, es indispensable el desarrollo de cursos ESP/EAP, aunque como se vio anteriormente, su éxito dependerá en gran parte de cómo las universidades fomentan la adquisición de una segunda lengua entre sus estudiantes y los orientan hacia la obtención de certificaciones internacionales que garanticen que estos se encuentren aptos para el desafío del inglés en ámbitos académicos o profesionales. Solo así los crecientes esfuerzos de las universidades por internacionalizarse se verán reflejados en los números de alumnos participantes $\mathrm{y}$, evidentemente, en la formación de personas preparadas para afrontar retos lingüísticos, culturales y profesionales nuevos. 


\section{REFERENCIAS}

Asamblea Nacional de Rectores e Instituto de Estadística e Informática (2010). II Censo Nacional Universitario. Lima, Perú: Dirección Nacional de Censos y Encuestas.

Castillo (2012). La Internacionalización de la Universidad Peruana. Lima, Perú: Asamblea Nacional de Rectores.

Crystal, David (2010). The Cambridge Encyclopedia of Language. Third Edition. UK. Cambridge University Press.

Ministerio de Educación (2009). Diseño Curricular Nacional de Educación Básica Regular. Lima, Perú: Ministerio de Educación.

Declaración de París de 2012 sobre los Recursos Educativos Abiertos. París, Francia: UNESCO. Recuperado de: http://www. unesco.org/new/fileadmin/MULTIMEDIA/HQ/CI/ $\mathrm{CI} /$ pdf/Events/Spanish_Paris_OER_Declaration.pdf

Delors, J. (1996). La Educación encierra un tesoro. Informe a la UNESCO de la Comisión Internacional sobre la Educación para el siglo XXI. París, Francia: Ediciones UNESCO.

Dumas, L. S. (1999). Learning a Second Language: Exposing Your Child to a new World of Words Boosts Her Brainpower, Vocabulary and Self-Esteem. Child, February 72, 74: 76-77.

Hyland, K. (2006). English for Academic Purposes. Oxon, England: Routledge.

International Association of Universities.(2014). Internationalization of Higher Education. Paris, France: UNESCO.
UNI incluyó por primera vez preguntas en inglés en su examen de admisión 2013. (Martes, 13 de agosto de 2013). Diario La República. Recuperado de: http:// www.larepublica.pe/13-08-2013/uni-incluyo-porprimera-vez-preguntas-en-ingles-en-su-examen-deadmision-2013

Kuder, M.; Lemmens, $\mathrm{N} \&$ Obst, D. (2014). Global Perspectives on International Joint and Double Degree Programs. German Academic Exchange Service \& Institute of International Education.

Jenkins, J. (2014). English as a Lingua Franca in the International University. Oxon, England: Routledge.

Paredes, F. (2014). Desmitificando la Traducción. Lima, Perú: Language and Academia. Recuperado de: http://www. languageandacademia.com/

Pontificia Universidad Católica del Perú, Dirección Académica de Relaciones Interinstitucionales (2010). Diagnóstico de la gestión e infraestructura de la cooperación internacional en universidades peruanas. Informe. Lima, Perú: Autor.

Programa Nacional de Becas y Crédito Educativo (2013). Memoria Anual 2013. Lima, Perú: Autor. Recuperado de: http://www.pronabec.gob.pe/inicio/publicaciones/ documentos/memoria2013.pdf

Salleres, M. (2013). [Entrevista no publicada con Mayra Yaranga]. 